\title{
Fracture Penetration Through an Interface
}

\author{
M.A. Biot \\ Consultant \\ W.L. Medlin \\ L. Massé \\ Mobil R\&D Corp.
}

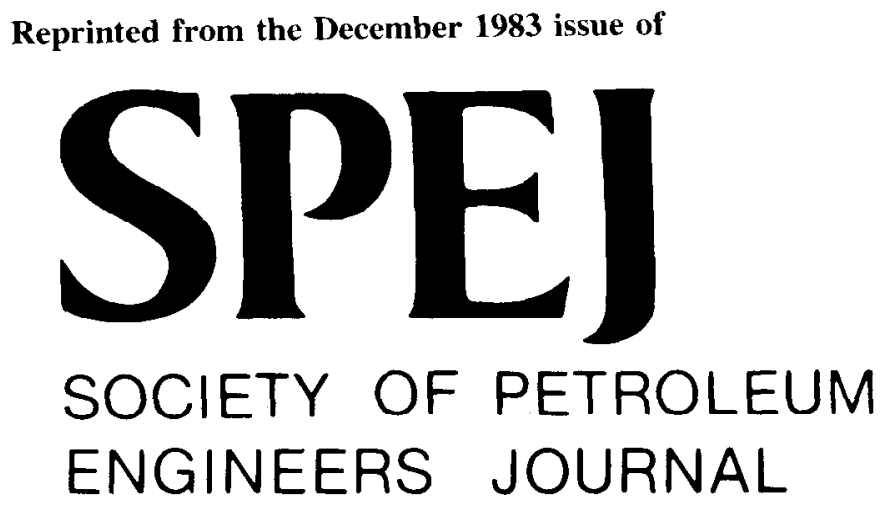




\section{Fracture Penetration Through an Interface}

M.A. Biot, consultant

W.L. Medlin, SPE, Mobil R\&D Corp.

L. Massé, Mobil R\&D Corp.

\begin{abstract}
This paper presents a combined theoretical and experimental investigation of fracture penetration through an interface. The results have application to hydraulicfracture containment in sandstone or limestone reservoir strata bounded by shale layers. Several simplifying assumptions and approximations are made. We assume that the interface separates dissimilar but adhering materials that are elastic to first order. We consider only differences in material properties and take stresses to be locally uniform. Approximations are made that reduce the fourth-order equation of plane strain elasticity to the second-order Laplace equation. Crack shape is taken to be sinusoidal and fluid leakoff is ignored.

Additional simplifying assumptions are made about the shape of the crack tip as it passes through the interface. Using a virtual work analysis, we derive a relation for internal fluid pressure required to extend the crack through the interface. This pressure is related to the equilibrium pressure needed to hold the crack barcly open. A simple relation is obtained involving only the shear modulus and surface energy of the materials on either side of the interface.

The theory was tested in laboratory experiments with Plexiglas ${ }^{\mathrm{TM}} /$ resin bonds. Test blocks of several configurations were used with various kinds of external loading. Cracks were initiated by applying grease pressure in thin notches. Pressures required to hold cracks in equilibrium were compared with those required to penetrate interfaces. Results were consistent with the theory within limits of precision in measuring surface energy. We conclude that the theory explains field observations of containment in a number of reservoirs that have been fractured hydrualically. However, practical value is limited by inability to estimate surface energies from logging or other wellbore data.
\end{abstract}

\section{Introduction}

Fracture penetration through an interface is of fundamental importance in hydraulic fracturing operations. The question of how far a fracture grows vertically during a fracturing treatment is basic to all design and optimization considerations. In a fairly homogeneous formation, such as granite, unlimited vertical growth might be expected. This would lead to a penny-shaped crack $^{1}$ of the kind expected for horizontal fractures. In layered, sedimentary rock, vertical growth requires penetration through interfaces between layers. If penetration is prevented at interfaces near the injection layer, the fracture will be contained and constant height thereafter.

Constant-height fracture geometries have been assumed in most theories of fracture propagation. ${ }^{2-6}$ Field results, notably temperature logs, have long indicated that this constant-height assumption is reasonable. Many of these logs can be explained only by the existence of some kind of barriers to vertical fracture propagation.

These considerations introduce the problem of crack penetration through an interface in a layered medium. This problem has received considerable attention already. It has been treated for both ideally elastic laminar materials and layered rock materials. Several studics have addressed the problem of a crack approaching an interface in a bonded-layer elastic material. ${ }^{9-11}$ These works have focused mainly on the nature of the singularity at the crack tip as it approaches the interface. The methods used have been based on the stress intensity concept introduced by Irwin. ${ }^{12} \mathrm{~A}$ similar approach has been used to treat the penetration problem in rock layers. Other studies have dealt with the fracture containment problem in reservoir rock by using the stress-intensity factor to evaluate penetration criteria. ${ }^{13-15}$

Our approach is much different. We use linear elasticity theory applied along the lines developed by Griffith ${ }^{16}$ for homogeneous materials. By introducing simplifying 


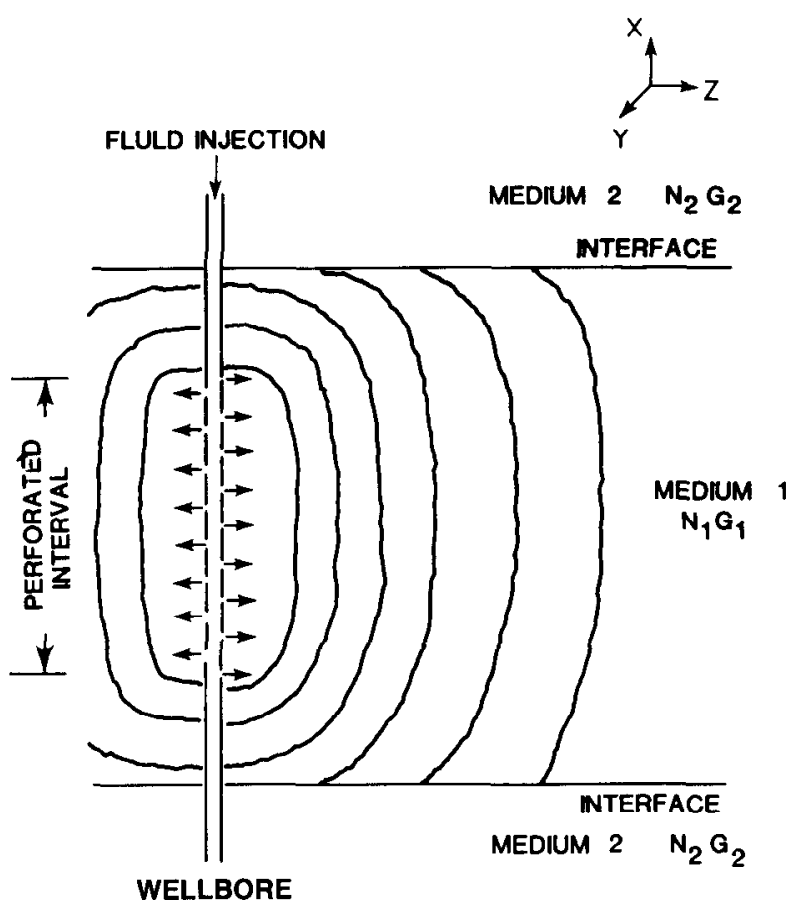

Fig. 1-Practical aspect of fracture penetration through an interface.

assumptions and approximations, we avoid the singularity problem and other difficulties associated with a heterogeneous medium. In this way we obtain a simple relation for predicting whether crack penetration will occur between bonded layers.

This approximate theory cannot serve the same purpose as the exact theory for a homogeneous medium. We have restricted our use of it to investigation of mechanical property differences only. We use relations derived from it to predict, in an approximate way, whether crack penetration will occur through an interface separating materials of dissimilar mechanical properties.

Recent investigations have indicated that stress differences between earth layers are an important source of fracture containment. ${ }^{17} \mathrm{We}$ do not address this problem here. Our approximate theory is not adequate to handle the problem of stress differences between layers. In our treatment the stress is taken to be equal in all layers, and, therefore, it does not appear in the results.

The various assumptions and approximations introduced into our theory must be justified. We have done this by means of direct experimental tests. The simple results predicted by our approximate theory can be definitively tested by laboratory experiments. We have devised tests using bonded Plexiglas and resin models to represent interfaces of the type considered in the theory. Laboratory tests of this kind have provided at least a qualitative verification of the approximate theory. Earlier experimental tests are also consistent with our theoretical predictions. ${ }^{18}$

\section{Theory}

We consider the practical field problem illustrated in Fig. 1. A hydraulic fracturing treatment is carried out through perforations or other means of fluid entry in a selected layer of reservoir rock. This layer, labeled Medium 1, is typically a brittle sandstone or limestone. As the fracture propagates both laterally and vertically, it approaches overlying and underlying interfaces with Medium 2, which has mechanical properties different from Medium 1. In typical cases, Medium 2 could be a shale. Using the coordinate system shown in Fig. 1, we take the boundaries between Media 1 and 2 to lie in the $y-z$ plane. We wish to know what conditions are required for penetration of the crack through the interface.

In our treatment, Media 1 and 2 are both taken to be orthotropic elastic materials with elastic symmetry about the $x$ and $y$ axes. We ignore leakoff of fluid through the crack faces. We also take fluid pressure in the crack to be uniform, ignoring the small variations that would result from fluid flow during crack propagation.

\section{Approximate Elasticity Theory}

We consider the plane-strain problem in the $x-y$ plane. The displacement vector field is denoted by $u$ and $v$ with strain components

$$
\begin{aligned}
& e_{x x}=\frac{\partial u}{\partial x}, \\
& e_{y y}=\frac{\partial v}{\partial y},
\end{aligned}
$$

and

$$
e_{x y}=\frac{1}{2}\left(\frac{\partial v}{\partial x}+\frac{\partial u}{\partial y}\right)
$$

The corresponding stress components are denoted by $\sigma_{x x}, \sigma_{y y}$, and $\sigma_{x y}$, respectively.

To reduce the problem to one that is tractable, we introduce certain approximations in the plane-strain analysis. We first assume that the stress produced by the crack in the direction of propagation is negligible-that is, $\sigma_{x x} \approx 0$. We also assume, along the same line of reasoning, that $\partial u / \partial y \ll \partial v / \partial x$. Appendix A shows that these approximations reduce the fourth-order biharmonic equation of classical theory for plane strain to the Laplace equation,

$$
\frac{\partial^{2} v}{\partial x^{2}}+\frac{\partial^{2} v}{\partial \eta^{2}}=0 \text {. }
$$

This allows us to represent the field $v$ by a harmonic potential function $\phi$ as

$$
v=\phi(x, \eta) \text {. }
$$

The stresses in terms of $\phi$ are then

$$
\sigma_{y y}=2 \sqrt{N G} \frac{\partial \phi}{\partial \eta}
$$

and

$$
\sigma_{x y}=G \frac{\partial \phi}{\partial x} .
$$




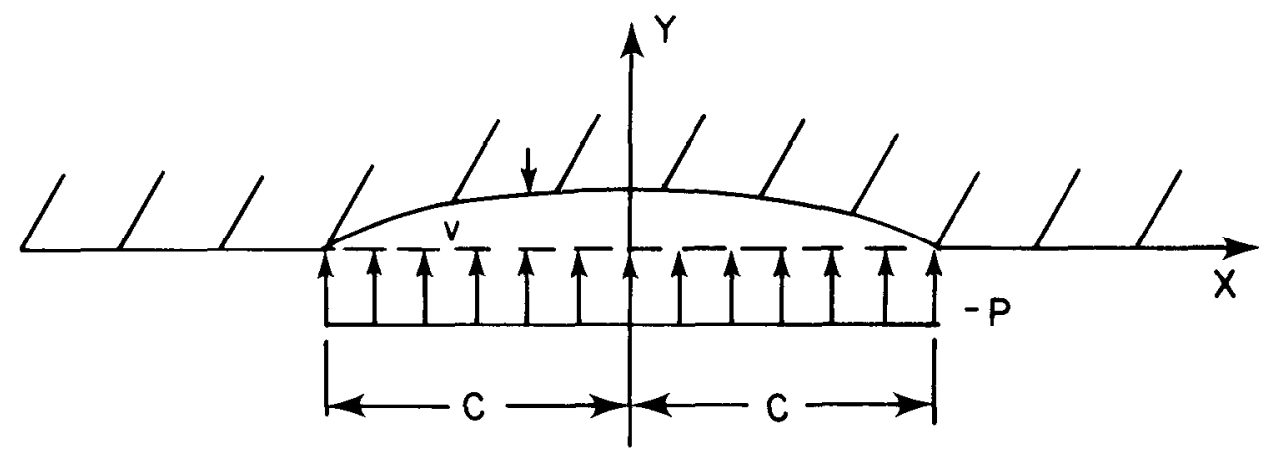

Fig. 2-Half-space representation of the crack.

Eqs. 2 through 5 provide the basis of an approximate theory for treating the crack-penetration problem. As a test of this theory, we have applied it in Appendix B to the classical Griffith crack problem ${ }^{16}$ illustrated in Fig. 2. In this case, it gives exact agreement with the classical result when the medium is incompressible (i.e., $v=1 / 2$ ). For $v<1 / 2$ the result is only approximate, with the error increasing as $v$ decreases.

\section{Sinusoidal Approximation}

Before treating the crack-penetration problem, we determine the conditions for crack propagation in Medium 1, which is taken to be isotropic. We consider a solution $\phi$, satisfying Eq. 2, which is sinusoidal along $x$, and given by

$$
\phi=B e^{-\ell \eta} \cos \ell x .
$$

This amounts to assuming a crack shape that is sinusoidal rather than elliptical. The crack length $2 c$ is taken to be the half wavelength of this sinusoidal shape as shown in Fig. 3; that is,

$$
\ell=\frac{\pi}{2 c} \text {. }
$$

A uniform pressure $p_{1}$ is now applied inside the crack. The sinusoidal solution (Eq. 6) can be used with the principle of virtual work to determine the approximate pressure $p_{1}$ required to extend the crack just a bit. The procedure is outlined in Appendix $C$. The approximate extension pressure obtained in this way is

$$
p_{1}^{2}=\frac{S_{1} \pi^{3}}{16 c} \sqrt{N_{1} G_{1}},
$$

where $S_{1}$ is the energy required to produce a unit area of crack surface in Medium 1.

Appendix $\mathrm{C}$ shows that a correction factor of 1.23 is required to bring this result into agreement with the one derived by Sneddon and Elliott ${ }^{19}$ for the classical Griffith problem.

\section{Penetration Through an Interface}

We consider now the problem of crack penetration as illustrated in Fig. 1. A crack of height $2 c$ is present in the perforated interval with elastic constants $N_{1}$ and $G_{1}$. The upper and lower tips of the crack are located at the two parallel interfaces nomal to the crack and separated by the distance $2 c$. The interfaces form boundaries with an outer material with elastic constants $N_{2}$ and $G_{2}$. Our purpose is to derive the conditions that determine whether the crack will propagate across the interface.

We locate the origin at one of the vertical tips of the crack and assume a sinusoidal shape

$$
v=B \sin P x,
$$

with $\ell$ given by Eq. 7 . We assume that, across $2 c$, this sinusoidal shape is still preserved after the crack penetrates the interface and extends into Medium 2 by a small amount.

Consider a small penetration into Medium 2 by a distance $b$ as shown in Fig. 4. Although the crack across $2 c$ is still sinusoidal, it has a new value of $\ell$ that must be determined. Eq. 9 corresponds to a function $\phi$ given by

$$
\phi=B e^{-\ell \eta} \sin \ell x \text {. }
$$

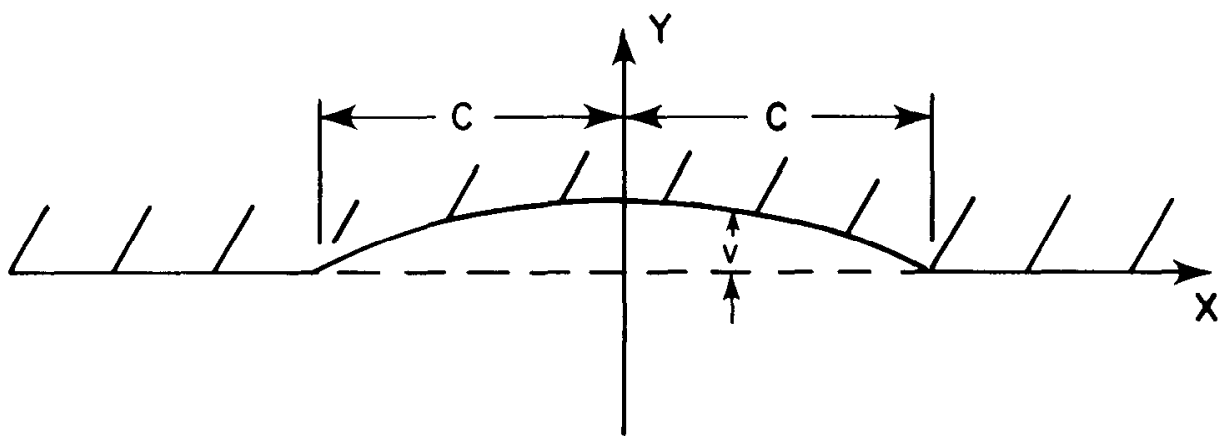

Fig. 3-Sinusoidal crack in the half space. 


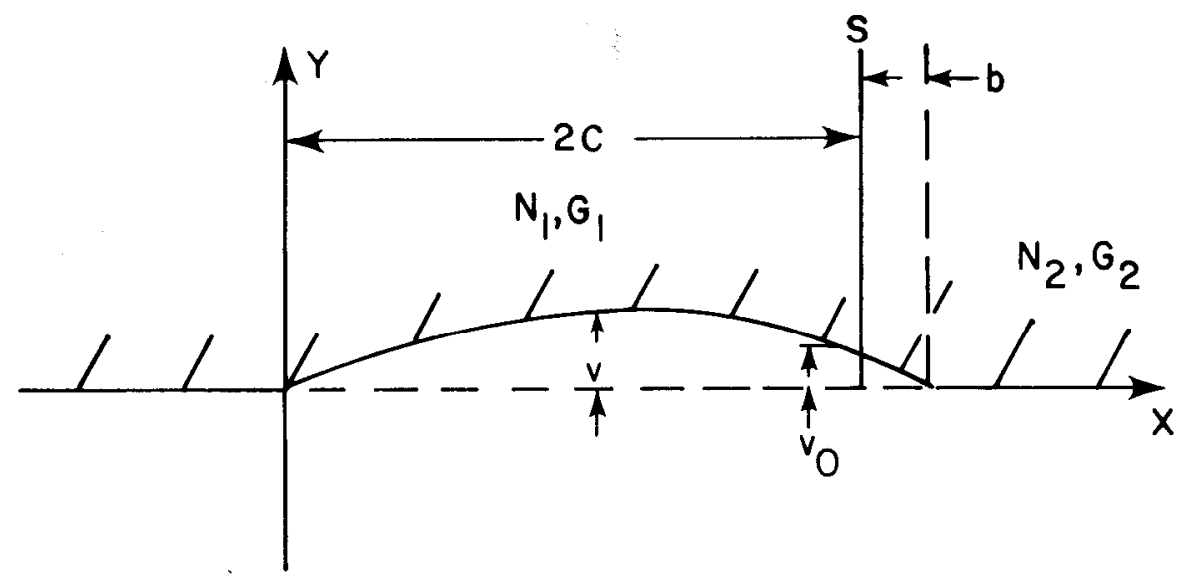

Fig. 4-Penetration of the crack across an interface $S$.

Also at the interface $x=2 c$, the shear stress given by Eq. 5 is

$$
\sigma_{x y}=G_{1} B \ell e^{-\ell \eta} \cos 2 \ell c .
$$

Along this interface, the displacement is

$$
\nu=B e^{-\ell \eta} \sin 2 \ell c \text {. }
$$

We now introduce another approximation. We replace Medium 2 by a layer of the same material of thickness $b$ and assume that this layer adheres to Medium 1 at $x=2 c$ and to a rigid base at $x=2 c+b$. The shear stress in this layer is given by

$$
\sigma_{x y}=-G_{2}(v / b)
$$

or

$$
\sigma_{x y}=-G_{2} \frac{B}{b} e^{-\ell \eta} \sin 2 \ell c
$$

Equating the shear stresses from Eqs. 11 and 14 gives

$$
\tan 2 \ell c=-\frac{G_{1}}{G_{2}} \ell b
$$

Since $b$ is small,

$$
2 \ell c \approx \pi-Z,
$$

where $Z$ is also small. This reduces Eq. 15 to

$$
Z=\frac{\pi b G_{1}}{2 c G_{2}}
$$

and gives the new value for $\ell$ in terms of $b$ as

$$
\ell=\frac{\pi}{2 c}\left(1-\frac{b G_{1}}{2 c G_{2}}\right) .
$$

If a fluid pressure $p_{2}$ is applied inside the crack, the condition for crack penetration can be determined in terms of $b$ from the energy balance equation

$$
p_{2} \frac{\partial V}{\partial b} \delta b-\frac{\partial W}{\partial b} \delta b=S_{2} \delta b
$$

Expressions for the crack volume, $V$, and the elastic energy, $W$, are derived in Appendix D. $V$ is given by

$$
V=\frac{32 c^{2} p_{2}}{\pi^{3} \sqrt{N_{1} G_{1}}}\left(1+\frac{b G_{1}}{2 c G_{2}}\right)^{2},
$$

and $W$ by

$$
W=\frac{16 c^{2} p_{2}{ }^{2}}{\pi^{3} \sqrt{N_{1} G_{1}}}\left(1+\frac{b G_{1}}{2 c G_{2}}\right)^{2} .
$$

Using Eqs. 20 and 21 in Eq. 19 gives

$$
p_{2}^{2}=\frac{S_{2} \pi^{3} \sqrt{N_{1} G_{1}}}{16 c\left(1+\frac{b G_{1}}{2 c G_{2}}\right)}\left(\frac{G_{2}}{G_{1}}\right)
$$

Since $b$ is small at the instant of penetration,

$$
\frac{b G_{1}}{2 c G_{2}} \ll 1
$$

and the penetration pressure is

$$
p_{2}{ }^{2}=\frac{S_{2} \pi^{3} \sqrt{N_{1} G_{1}}}{16 c}\left(\frac{G_{2}}{G_{1}}\right) .
$$

Eq. 23 can be compared with Eq. 8 for $p_{1}$, the pressure required to propagate the crack in Medium 1. The ratio is

$$
\frac{p_{2}}{p_{1}}=\sqrt{\frac{S_{2} G_{2}}{S_{1} G_{1}}} .
$$


Eq. 24 gives the condition required for penetration of the crack through the interface. If

$$
S_{2} G_{2}<S_{1} G_{1}, \ldots \ldots \ldots \ldots \ldots \ldots \ldots \ldots
$$

penetration will occur. If

$$
S_{2} G_{2}>S_{1} G_{1} \text {, }
$$

the crack will be confined to Medium 1. In this case, penetration will occur only if the pressure is increased by the ratio given by Eq. 24 .

The absolute pressures given by Eqs. 8 and 23 are approximate because of the many simplifications required in the theory. However, correction factors for both equations should be about the same. Therefore, errors in the ratio should be small.

An additional result that tends to confirm the analysis is obtained by letting the interface disappear. If we take $S_{2}=S_{1}$ and $G_{2}=G_{1}$, Eq. 22 reduces to

$$
p_{1}{ }^{2}=\frac{S_{1} \pi^{3} \sqrt{N_{1} G_{1}}}{16(c+b / 2)}
$$

This is the same result obtained by replacing $c$ with $c+b / 2$ in Eq. 8.

\section{Experimental Verification}

The results predicted by Eqs. 24 through 26 have been tested by means of simple lab experiments. One type of experiment is illustrated in Fig. 5. Blocks made of bonded dissimilar matcrials werc prepared in the form shown. The materials used were Plexiglas and a hard brittle resin with the trade name Stycast.

Blocks were made from either Plexiglas or resin as the inner Medium 1. When Plexiglas was used, the outer Stycast was bonded to a $2.5 \times \times 4 \times 4$-in. [ $6.35 \times 10 \times$ $10-\mathrm{cm}]$ milled Plexiglas block by use of a form for pouring the initially mixed resin and hardener. After setting up, the resin faces were milled to the final compound block dimensions, $2.5 \times 6 \times 7$ in. [6.35 $\times 15.24 \times 17.78$ $\mathrm{cm}]$. When the materials were reversed, the blocks were made by milling the outer Plexiglas part and filling in the inner Stycast by pouring the resin into a form. The resin faces were then milled to the proper dimensions after setting up.

The inner material was notched for fracture initiation. The notch was a slot $0.010 \mathrm{in} .[0.254 \mathrm{~cm}]$ wide $\times 0.30$ in. $[0.762 \mathrm{~cm}]$ deep $\times 2.5 \mathrm{in} .[6.35 \mathrm{~cm}]$ long cut with a slitting saw. It was centered in the $2.5 \times 4$-in. $[6.35 \times 10-\mathrm{cm}]$ face parallel to the $4-\mathrm{in}$. [10-cm] edge as shown in Fig. 5. The walls of this notch contained small brass capacitor plates for use in measuring crack width during propagation. These plates were formed by initially milling a recess $0.55 \mathrm{in}$. $[1.40 \mathrm{~cm}]$ in diameter by 0.25 in. $[0.635 \mathrm{~cm}]$ deep in the center of the face. A $0.50 \times 0.25$-in. [1.27 $\times 0.635-\mathrm{cm}]$ brass disk was epoxied into this recess. The solid disk was cut in half by the slitting saw during the notch-cutting operation. The half disks served as plates of a capacitor that was connected by soldered wire leads to a capacitance bridge. The bridge measurements gave the spacing between the plates during crack growth, which served as a measure of crack width at the crack entrance.
The Plexiglas/resin block was mounted in a servocontrolled hydraulic press to apply a small stress on the end face containing the notch and its opposite face. A second hydraulic press was positioned inside the posts of the servo press to apply a small stress to the other pair of end faces perpendicular to the notch. No stress was applied to the remaining side faces, which were parallel to the plane of the notch. These conditions favored propagation of the crack in the plane of the notch.

The press platen bearing against the notched face included an injection system for injecting grease into the slot. This platen contained an O-ring groove that was rectangular with rounded corners. A standard O-ring was used in this groove to form a seal with the milled sample block face. This seal extended past the Plexiglas/resin interface far enough to maintain sealing during crack propagation in the Plexiglas.

Grease was injected into the notch by means of a modified Ruska pump connected to the injection system through stainless-steel high-pressure tubing. The crack width was monitored by recording an error signal from the capacitance bridge. Details of this technique are given elsewhere. ${ }^{20}$ The crack length was observed visually and recorded in some cases by a motion picture camera. The injection pressure was measured by a transducer mounted on the injection plate.

This arrangement provides a simple experimental model for testing the theoretical predictions of the preceding section. A test of Eqs. 24 through 26 required values of the shear modulus, $G$, and surface energy, $S$, for the Plexiglas and resin materials. These quantities were determined by established methods.

The shear modulus was determined from stress/strain data by strain-gauge techniques. Cylinders of 1-in. [2.54-cm] diameter and 2-in. [5.08-cm] length were machined from blocks of the Plexiglas and resin materials used in the crack propagation experiments. Standard SR4-type 120- $\Omega$ gauges were bonded to the surface of each cylinder with Eastman $910^{\mathrm{TM}}$ adhesive. We used prefabricated right-angle patterns for simultaneous measurement of longitudinal and circumferential strains. Gauge patterns were mounted on opposite faces centered on the cylinder length. Strains were measured with Daytronic Corp. Type 90 input modules in transducer conditioner panels. An aluminum cylinder was used as a standard to check gauging procedures, sensitivity settings, etc.

The stress/strain data were obtained by applying step loads of approximately 250,500 , and $1,000 \mathrm{lbm}[113$, 218 , and $454 \mathrm{~kg}$ ] to each cylinder. Over this range the longitudinal and circumferential strains were linear with stress. However, under each step-load application, the strain responses for both Plexiglas and resin cylinders were characteristic of slightly viscoelastic materials. The aluminum standard showed purely elastic response under the same conditions.

The elastic constants were obtained from the relations

$$
\begin{aligned}
& E=\frac{\sigma_{z}}{\epsilon_{z}}, \\
& \mathbf{v}=\frac{\epsilon_{\theta}}{\epsilon_{z}},
\end{aligned}
$$




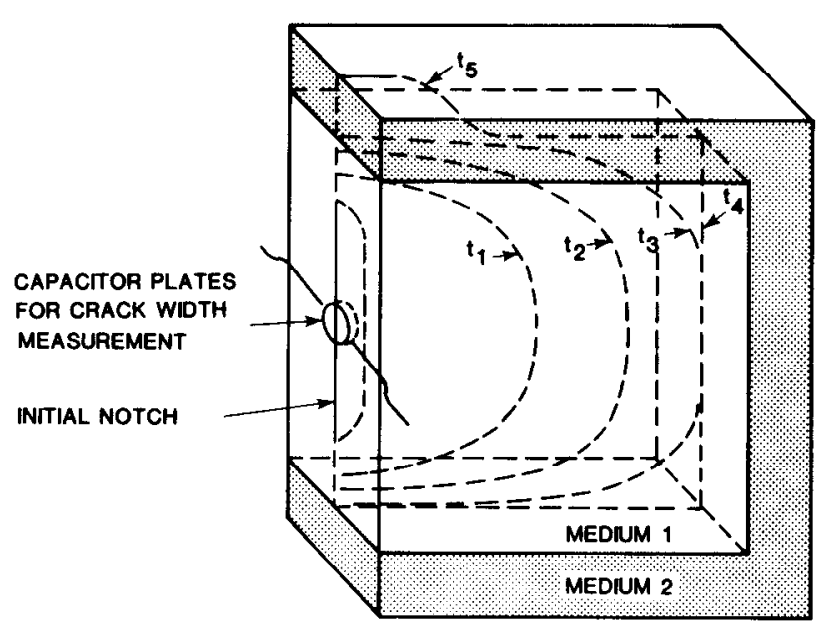

Fig. 5-Experimental test of crack penetration across an interface.

and

$$
G=\frac{E}{2(1+v)} .
$$

Results for the Plexiglas and resin materials are given in Table 1. These results are averages of five sets of measurements with standard deviations as given.

The surface energies were determined by a method described by Gilman. ${ }^{21}$ This is only one of several methods described in the literature for measuring surface energy. ${ }^{22-24}$ We chose it over others because it is simple and direct and, for our purposes, gave acceptable reproducibility of results.

Fig. 6 illustrates our experimental arrangement. Cleavage bars with the indicated dimensions were milled from the Plexiglas and resin blocks used in the fracturing experiments of Fig. 5. A servo-controlled hydraulic press was used to load the bars in tension. Pins mounted in the end holes were connected through swivels between the movable ram and a load cell mounted on the press frame. The ram was moved at constant rate by using a ramp-controlled linear variable displacement transformer (LVDT) as the sensor for the servo system. The same LVDT was used to record displacement, $\delta$.

A sharp blade was used to cut a notch in the 0.125 -in.[0.3175-cm-] wide face in which crack growth started. A tensile load $T$ was applied at a rate of 300 to $500 \mathrm{~g} / \mathrm{s}$ until cleavage of the bar began. The load was then immediately reduced to zero. This usually produced an initial crack $1 / 2$ to 1 in. [ 1 to $2 \mathrm{~cm}$ ] long corresponding to $L \approx 1 \frac{1}{2}$ to 2 in. [ 4 to $5 \mathrm{~cm}$ ]. This $L$ value was measured and the tensile load was again applied at the same rate as before. The tensile load at which crack growth started a second time was determined and recorded as $F$. These values of $F$ and $L$ were used with the Gilman equation, ${ }^{21}$

$$
S=\frac{6 F^{2} L^{2}}{Y w^{2} h^{3}}
$$

to compute the surface energy $S$.

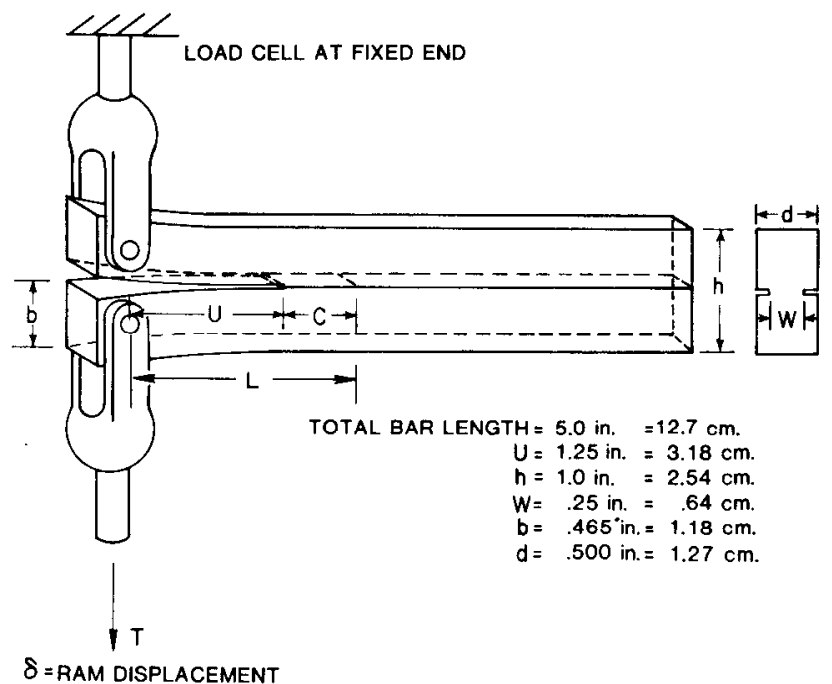

Fig. 6-Experimental arrangement for determining surface energy.

In resin samples, the appropriate value of $F$ was well defined as illustrated in Fig. 7a. The second loading of the initial crack produced a sharp break in $T$ that usually propagated the crack to the end of the bar. In Plexiglas samples, $F$ was less well defined because of a tendency of the crack to grow out of the slotted plane of width $w$. The cause is not clear but may be associated with residual strains induced during fabrication. Typically, after departing from the slot the crack traveled slowly over a curved surface and bent back into the slotted plane, usually over a distance of $1 / 2$ to $1 \mathrm{in}$. [1 to $2 \mathrm{~cm}$ ]. This deviation produced results like those shown in Fig. $7 b$.

The crack growth outside the slotted plane clearly required higher tensile forces than growth in the plane. However, this presumably would be of little consequence if the initial break in $F$ occurred while the crack was in the proper plane. We rejected all measurements where this was clearly not the case; nevertheless, our results were probably influenced by this effect in most measurements. Thus, $F$ values for Plexiglas are likely to be too large, on the average, and surface energies in error on the high side.

The values for $Y$ in Eq. 26 were determined from three-point bending measurements on bars of the resin and Plexiglas materials. These values were found to be in good agreement with $E$ values from strain-gauge measurements.

Table 1 gives deflection data from repeated measurements and the surface energies computed from them using Eq. 28. Standard deviations in the results are also given. The surface energy of the resin is considerably larger than that of Plexiglas. However, the shear moduli are, unfortunately, about the same. Therefore, while experiments with these materials provide a good test of surface energy effects, they give little information about sensitivity to shear modulus.

Applying the results of Table 1 to the experiment of Fig. 5 for the case where Medium 1 was Plexiglas and Medium 2 was resin, we have

$$
S_{2} G_{2}=1.54 S_{1} G_{1}
$$



PENETRATION EXPERIMENTS

\begin{tabular}{|c|c|c|c|c|}
\hline Material & $\begin{array}{c}E \\
\left(\mathrm{~Pa} \times 10^{9}\right)\end{array}$ & $v$ & $\begin{array}{c}G \\
\left(\mathrm{~Pa} \times 10^{9}\right)\end{array}$ & $\begin{array}{c}S \\
\left(\mathrm{Nm} / \mathrm{m}^{2}\right)\end{array}$ \\
\hline $\begin{array}{l}\text { Plexigla } \\
\text { Resin }\end{array}$ & $\begin{array}{l}3.01 \pm 0.01 \\
2.69 \pm 0.01\end{array}$ & $\begin{array}{l}0.341 \\
0.359\end{array}$ & $\begin{array}{l}1.12 \pm 0.01 \\
0.986 \pm 0.002\end{array}$ & $\begin{array}{l}2.78 \pm 0.37 \\
4.85 \pm 0.36\end{array}$ \\
\hline
\end{tabular}

In this case our theory predicts that a crack initiated in the Plexiglas and propagated at a slow rate should stop at the resin interface and remain stationary until the pressure has increased by the ratio

$$
\frac{p_{2}}{p_{1}}=\sqrt{1.54}=1.24
$$

At the pressure $p_{2}$, breakthrough should occur into the resin.

To test these predictions, we conducted fracturing tests in several of the models of Fig. 5 with Plexiglas as Medium 1. Results from a typical experiment are presented in Fig. 8. Here we show crack width and injection pressure, plotted as a function of time, at a grease injection rate of $0.5 \mathrm{~mL} / \mathrm{min}\left[0.5 \mathrm{~cm}^{3} / \mathrm{min}\right]$. The times labeled $t_{1} \ldots t_{5}$ correspond to the crack propagation profiles sketched in Fig. 5.

Qualitatively, the events in Fig. 8 are in striking agreement with theoretical predictions. As shown in Fig. 5, following initiation, the crack grew both laterally and vertically through time $t_{3}$ to reach the resin interfaces on each of the Plexiglas faces. During this period, the crack width grew slightly, and the injection pressure declined slowly.

At $t_{3}$, the injection pressure had become almost constant at a value we call $p_{1}$. Since crack growth was so slow, $p_{1}$ is only a little higher than the static pressure of Eq. 8 required to extend the crack a fraction.

Following $t_{3}$, the crack moved to fill out the square profile of the Plexiglas and then remained stationary at the interface. At this time the injection pressure began to increase and the crack width grew at a much higher rate. At $t_{5}$, after the injection pressure had more than doubled, the crack broke through the interface.

On a quantitative basis, the $p_{2} / p_{1}$ ratio is considerably larger than predicted. The average for all measurements was 2.0. However, considering the many approximations in the theory and the less-than-ideal nature of the experiments, this is not surprising. The theory is derived for plane strain, whereas the experiments produced a 3D crack. In the theory, we assume ideal elastic behavior, but the Plexiglas and resin materials are slightly viscoelastic. The theory is exact only for incompressible materials (i.e., for $v=1 / 2$ ). In the experimental materials we had $v \approx 0.35$. Our measurements of $S$ for Plexiglas and for the static pressure $p_{1}$ are both considered to be in error on the high side, consistent with the discrepancy here.

Additional experiments were conducted by reversing the materials so that the crack was initiated in the resin that served as Medium 1. In this case, the crack propagated through the interface into the Plexiglas with almost no delay or pressure increase as predicted.
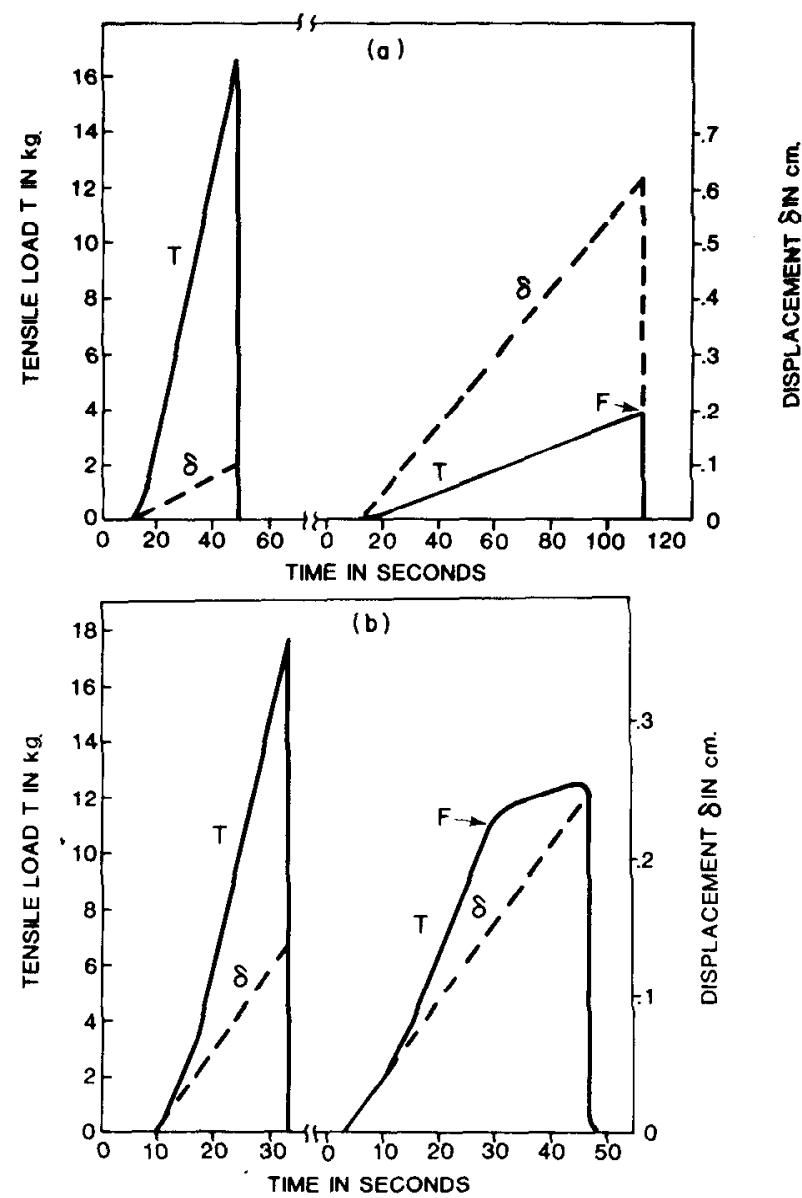

Fig. 7-Load-deflection curves for typical surface energy determinations in (a) resin, (b) Plexiglas.

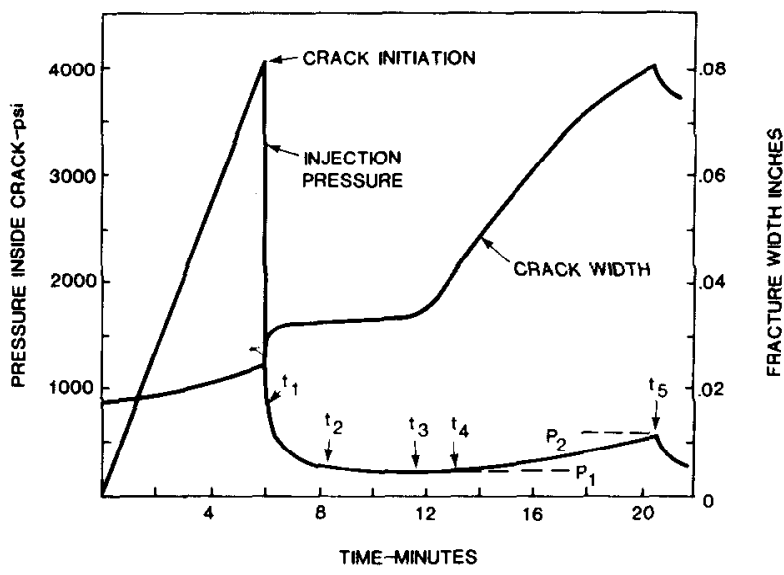

Fig. 8-Experimental data obtained in a test with the arrangement of Fig. 6 using Plexiglas as Medium 1 and resin as Medium 2 


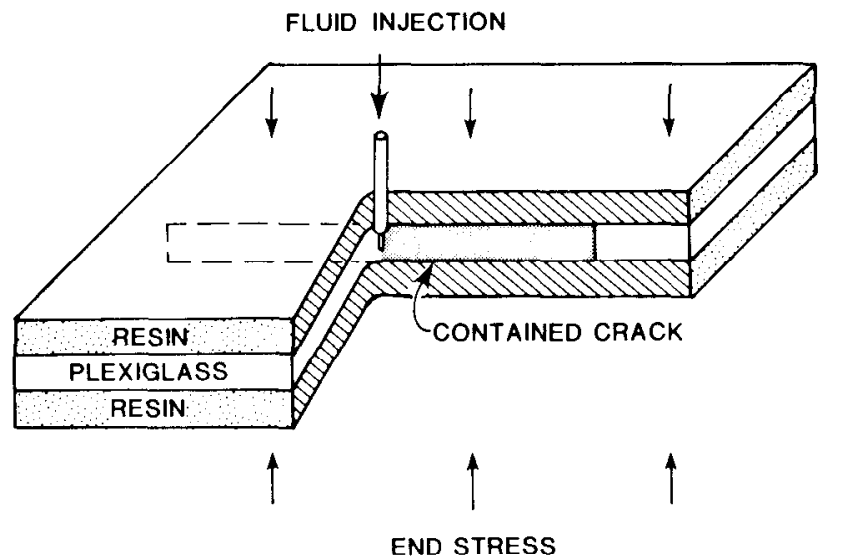

Fig. 9-Experimental arrangement for testing fracture containment with free lateral crack extension.
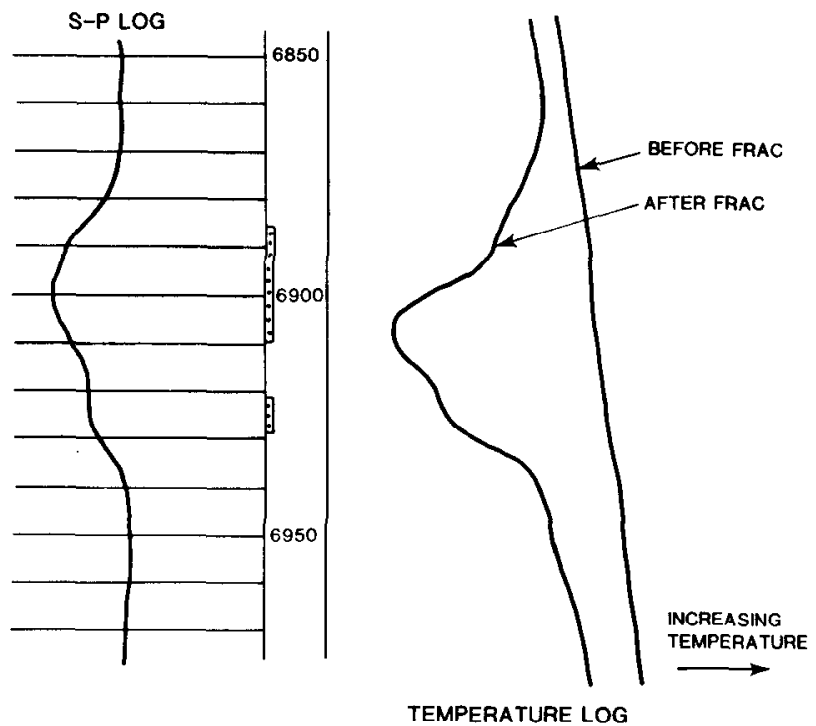

Fig. 10-Temperature log illustrating fracture containment in field treatment.
A second type of experiment using the same Plexiglas and resin materials was conducted with the arrangement shown in Fig. 9. To make the model illustrated here, a Plexiglas plate 2 in. [5.1 cm] thick and 30 in. [76.2 cm] along each edge was bonded to resin layers 2 in. [5.1 $\mathrm{cm}$ ] thick. A steel tube was epoxied into a hole drilled through the center of the upper resin layer 0.75 in. [1.9 $\mathrm{cm}]$ into the Plexiglas. A hole 0.125 in. $[0.3175 \mathrm{~cm}]$ in diameter was drilled past the tube into the Plexiglas to within $0.75 \mathrm{in} .[1.9 \mathrm{~cm}]$ of the opposite resin face. This layered block was loaded in a hydraulic press between steel plates bearing against the $30 \times 30$-in. $[76.2 \times 76.2-\mathrm{cm}]$ resin faces. A stress of a few psi was applied to these faces. Grease was injected into the borehole at constant rate. This produced initiation of a vertical crack in the Plexiglas at the borehole. The crack grew both laterally and vertically until it reached the resin interfaces. At this point the vertical extension stopped and the crack grew laterally, as indicated in Fig. 9, to the edges of the Plexiglas plate. This plastic model is representative of the mode of propagation that can be expected in many reservoir intervals bounded by shales or other lithologically different materials.

These experimental results confirm the most important basic features of the theory. They show that mechanical property differences alone are sufficient to prevent fracture penetration through a bonded interface provided fluid pressure in the crack remains below a critical value. They confirm that surface energy is one of the important mechanical properties controlling penetration. They also show that fluid pressure in the crack is critically involved in the penetration. If conditions favor containment of the crack at an interface, penetration will not occur until the critical pressure is exceeded. Under normal conditions of brittle crack propagation, if the crack does not penetrate at an interface on initial contact, it will not do so later. The results further show that an interface that favors containment for crack approach from one direction does not impede penetration from the opposite direction.

Although our experiments demonstrated the impor- tance of surface energy in controlling penetration, they have not confirmed the importance of shear modulus. Additional tests with materials having much different shear moduli but roughly equal surface energies would have been desirable. However, time restrictions prevented additional work along these lines.

Our experimental results with plastic models are consistent with results reported by Daneshy ${ }^{6}$ for rock materials bonded with adhesive. Surface energy and shear modulus were not measured in these experiments. However, from fracturability tests, which were included, it can be inferred that the conditions for penetration were at least qualitatively consistent with Eq. 24.

\section{Field Applications}

There are numerous examples in field experience where fracturing results are consistent with the equations derived herc. Hydraulic-fracturing treatments are frequently carried out in a zone of one lithology overlain by a zone of different lithology bonded along a reasonably sharp interface. Sandstone or limestone intervals overlain by shales are the most common example. Temperature logs obtained before and after fracturing in such cases frequently show that the fracture did not penetrate into the overlying zone.

An example of such a case is shown in Fig. 10. This figure shows temperature logs run before and after a very large fracturing treatment in a $50-\mathrm{ft}[15-\mathrm{m}]$ sandstone interval. The accompanying spontaneous potential (SP) $\log$ shows a fairly sharp interface between the sandstone and a thick overlying shale. The temperature logs indicate that there was no penetration of the fracture into the main shale zone. Such temperature logs are subject to various interpretations. ${ }^{25}$ However, it is difficult to make a case for vertical fracture growth through the shale in this case. We find the example of Fig. 10 to be typical of much of our fracturing experience.

The theory developed here can explain Fig. 10 and other examples like it, on the basis of mechanical property differences between the two zones. This is not to say 
that stress conditions do not also play an important role. But stress differences are not required to explain fracture containment or the absence of it in a given case.

Our theoretical results are of little value in predicting fracture penetration in specific field cases. This is because of the difficulties in determining surface energy and shear modulus in earth materials. Such determination would be limited to laboratory measurements in core samples. Even these would be restricted to competent rock intervals. Laboratory measurements in materials such as shales, for example, would have little meaning because of the changes induced by removing the material from the earth. In-situ measurements are also impractical. In-situ methods of measuring surface energy are unknown and it is difficult to conceive of logging or seismic data that could yield this quantity. In-situ determinations of shear modulus from sonic logs are not impractical. However, since rocks are not ideally elastic, there is no assurance that the dynamic modulus from a sonic $\log$ would be even approximately equal to the required static value.

The value of the theory is not in predicting results for specific cases but in establishing a general principle. The example of Fig. 10 is not uncommon in fracturing applications. Therefore, it can be assumed that, in many field cases, barriers will exist above and below the productive interval that will prevent vertical fracture penetration at treating pressures below the limit given approximately by Eq. 24 . When this is the case, a hydraulically induced fracture will extend vertically to the barriers and stop, as in the plastic model experiment of Fig. 9. At this point, under normal conditions of brittle crack growth, fluid pressure in the fracture will be declining, or, at worst, remaining roughly constant. Therefore, if fracture penetration did not occur at the barrier initially, it will not occur later, as long as the injection rate is not increased. This means that the fracture will maintain a constant height and grow only laterally away from the wellbore as in the plastic model of Fig. 9. This is one of the simplifying assumptions made in most theories of fracture propagation. The results presented here show that this assumption is reasonable.

These results should be considered in planning largescale fracturing operations in fields containing many wells. The potential barriers to vertical fracture extension must be guessed at initially. But fracturing experience in the first wells to be treated can be used to identify the barriers if they exist. Provisions should be made to extend the temperature logs well below the treated interval to identify the underlying as well as the overlying barrier. The interval determined in this way can then be taken as the expected fracture height for design of subsequent treatments in the field.

Fracturing-mineback experiments reported by Warpinski et al. ${ }^{17}$ have important bearing on our results. These experiments, conducted at the Nevada Test Site, showed that fractures generated in an ash-fall tuff zone penetrated into an overlying welded tuff having considerably higher shear modulus. Surface energies were not measured. However, modulus values, together with physical descriptions, suggest that the welded tuff should have higher surface energy than the ash fall. Therefore, penetration of the fracture into the welded tuff seemingly contradicts Eq. 26. However, the transition between zones is not sharp in this case but consists of several feet of highly varied lithology. Consequently, it is not surprising that our theory is not applicable in this case.

The mineback results emphasize an important point regarding application of our theory. The nature of the interface between dissimilar rock materials is an important factor. The limits of applicability of our simplified theory are difficult to assess in this regard. The welldefined interfaces of our Plexiglas resin models and Daneshy's cemented rock models ${ }^{18}$ will not be found very often in reservoir rock. On the other hand, the interfaces typically exposed in outcrops, roadcuts, and other excavations suggest that sharp transitions between lithologically different rock layers are not rare. However, the applicability of our theory probably depends more on this factor than any other.

\section{Conclusions}

An approximate theory has been developed for fracture penetration through an interface between bonded dissimilar materials under uniform stress. The theory shows that mechanical property differences on either side of such an interface can prevent fracture penetration. The product of surface energy and shear modulus is shown to be the dominant factor controlling penetration. When this product favors containment, penetration will not occur until fluid pressure in the crack exceeds a critical value. The critical pressure is related in a simple way to the surface energy/shear modulus products for materials on either side of the interface

The basic features of this theory have been confirmed by laboratory experiments in plastic models. These experiments verify that mechanical property differences are sufficient to prevent fracture penetration until a critical fluid pressure is exceeded in the crack. They also confirm that surface energy is one of the controlling mechanical properties. However, the experiments did not prove that shear modulus is the other controlling factor.

The theory developed here is of little benefit in predicting fracture containment or penetration in specific field cases. Problems in determining surface energy and shear modulus preclude this use of the theory. Its main value is in establishing a general principle with broad field applications. Under normal conditions of brittle fracture propagation, penetration of an interface must occur when the crack makes initial contact. Normal decline in fluid pressure in the crack prevents penetration at a later time. In field operations, this can lead to fractures that have constant height throughout most of a fracturing treatment

This concept should be applied in large field operations involving fracturing treatments in many wells. Temperature logs should be used in initial treatments to recognize interfaces that provide fracture containment. If such interfaces exist, they can be assumed to determine a constant fracture height which can be used for design and optimization purposes.

\section{Acknowledgments}

We thank R.E. Aikin and K.L. Sims for assistance in the experimental measurements and Mobil R\&D Corp. for permission to publish this paper. 
$A=$ constant of integration in solution

(Eq. B-5) of Laplace's

equation (Eq. 2) in polar

coordinates

$b=$ penetration distance of crack into

Medium 2 as illustrated in Fig. 4

$B=$ constant of integration in sinusoidal solution (Eq. 6) of Laplace's equation (Eq. 2) in $x-y$ plane

$2 c=$ crack length from tip to tip

$e_{x x}=x$ strain component

$e_{y y}=y$ strain component

$e_{x y}=x-y$ strain component

$E=$ Young's modulus, dyne $/ \mathrm{cm}^{2}[\mathrm{~Pa}]$

$F=$ tensile force on bar of Fig. 6 at which crack propagation is initiated, dyne [mN]

$G=$ shear modulus, dyne $/ \mathrm{cm}^{2}[\mathrm{~Pa}]$

$h=$ thickness of bar of Fig. 6 , in. [cm]

$L=$ crack length in bar of Fig. 6 at which crack propagation is initiated, in. [cm]

$\ell=$ inverse length defined by Eq. 7 .

$N=$ elastic constant appearing in Eq. 4

$p=$ internal fluid pressure in crack, dyne $/ \mathrm{cm}^{2}[\mathrm{~Pa}]$

$r=$ polar coordinate

$S=$ surface energy, $\mathrm{erg} / \mathrm{cm}^{2}\left[\mathrm{Nm} / \mathrm{m}^{2}\right]$

$t=$ time variable

$T=$ tensile load applied to cleavage bars of Fig. 6, dyne [mN]

$u, v=$ displacement vectors

$v_{o}=$ crack displacement at interface

$V=$ crack volume

$w=$ width of bar of Fig. 6 across notched region, in. [cm]

$W=$ energy required to generate crack volume $V$

$x, y=$ space coordinates

$Y=$ Young's modulus in beam bending

$z=$ complex quantity defined by Eq. B-3

$Z=$ dimensionless quantity defined by Eq. 16

$\delta=$ displacement

$\epsilon_{z}=$ longitudinal strain measured in cylindrical specimens by strain gauge techniques

$\epsilon_{\theta}=$ circumferential strain measured in cylindrical specimens by straingauge techniques

$\zeta(r, \theta)=$ function used to represent solution of Laplace's equation (Eq. 2) defined by Eq. B-4

$\eta=$ space coordinate defined by Eq. 3

$\theta=$ polar coordinate

$v=$ Poisson's ratio

$\sigma_{x x}, \sigma_{y y}, \sigma_{x y}=$ stress components drical specimen for strain-gauge

measurements, dyne $/ \mathrm{cm}^{2}[\mathrm{~Pa}]$

$\phi(x, \eta)=$ harmonic potential function used to represent displacement vector $v$

\section{References}

1. Sneddon, I.N.: "The Distribution of Stress in the Neighborhood of a Crack in an Elastic Solid,' Proc. Roy Soc. of London, A187 (1946) 229-60.

2. Khristianovitch, S.A. and Zheltov, Y.P.: "Formation of Vertical Fractures by Means of Highly Viscous Liquid," Proc., Fourth World Pet. Cong., Rome (1955) Sec. 11, 579-86.

3. Perkins, T.K. and Kern, L.R.: "Widths of Hydraulic Fractures," J. Pet. Tech. (Sept. 1961) 937-49.

4. Nordgren, R.P.: "Propagation of a Vertical Hydraulic Fracture," Soc. Pet. Eng. J. (Aug. 1972) 306-14.

5. Geertsma, J. and de Klerk, F.: "A Rapid Method of Predicting Width and Extent of Hydraulically Induced Fractures," J. Pet. Tech. (Dec. 1969) 1571-81.

6. Daneshy, A.A.: "On the Design of Vertical Hydraulic Fractures," J. Pet. Tech. (Jan. 1973) 83-97.

7. Bogy, D.B.: "On the Plane Elastostatic Problem of a Crack Terminating at a Material Interface,' J. Appl. Mech. (Dec. 1971) 38, 911-18.

8. Hilton, P.D. and Sih, G.C.: "A Laminate Composite With a Crack Normal to the Interfaces," Intl. J. Solids Structures (1971) 7, 913-30.

9. Cook, T.S. and Erdogan, F.: "Stresses in Bonded Materials With a Crack Perpendicular to the Interface," Intl. J. Eng. Sci. (1972) 10, 677-97.

10. Erdogan, F. and Biricikoglu, V.: "Two Bonded Half Planes With a Crack Going Through the Interface,"' Intl. J. Eng. Sci. (1973) 11, 745-66.

11. Comninnu, M. and Dundurs, J.: "A Closed Crack Terminating at an Interface," J. Appl. Mech. (March 1979) 46, 97-100.

12. Irwin, G.R.: "Analysis of Stresses and Strains Near the End of a Crack Traversing a Plate," J. Appl. Mech. (Sept. 1957) 24. 361-64.

13. Simonson, E.R., Abou-Sayed, A.S., and Clifton, R.J.: "Containment of Massive Hydraulic Fractures," Soc. Pet. Eng. J. (Feb. 1978) 18, 27-32.

14. Cleary, M.P.: "Primary Factors Governing Hydraulic Fractures in Heterogeneous Stratified Porous Formations," paper ASME 78-Pet-47 presented at the 1978 ASME Energy Technology Conf., Houston.

15. van Eekelen, H.A.M.: "Hydraulic Fracture Geometry: Fracture Containment in Layered Formations," Soc. Pet. Eng. J. (June 1982) 22, 341-49.

16. Griffith, A.A.: "The Theory of Rupture," Proc., Intl. Cong. Appl. Mech., Delft (1924) 55-63.

17. Warpinski, N.R., Schmidt, R.A., and Northrop, D.A.: "Laboratory Investigation of the Effect of In-Situ Stresses on Hydraulic Fracture Containment," Soc. Pet. Eng. J. (June 1982) 333-40.

18. Daneshy, A.A.: "Hydraulic Fracture Propagation in Layered Formations," Soc. Pet. Eng. J. (Feb. 1978) 33-41.

19. Sneddon, I.N. and Elliott, H.A.: "The Opening of a Griffith Crack Under Internal Pressure,' Quart. Appl. Math. (1946) 4, 262-67.

20. Medlin, W.L. and Massé, L.: "Laboratory Experiments in Fracture Propagation," paper SPE 10377 presented at the 1981 SPE Regional Meeting, Columbus, OH, Nov. 4-6.

21. Gilman, J.J.: "Direct Measurements of the Surface Energies of Crystals," J. Appl. Phys. (Dec. 1960) 31, 2208-18.

22. Berry, J.P.: "Determination of Fracture Surface Energies by the Cleavage Technique,'” J. Appl. Phys. (Jan. 1963) 34, 62-68.

23. Perkins, T.K. and Bartlett, L.E.: "Surface Energies of Rocks Measured During Cleavage," Soc. Pet. Eng. J. (Dec. 1963) 307-13.

24. Partington, J.R.: "An Advanced Treatise on Physical Chemistry," Longmans, Green \& Co., London (1952) 3, 242.

25. Dobkins, T.A.: "Improved Methods To Determine Hydraulic Fracture Height," J. Pet. Tech. (April 1981) 719-26.

26. Biot, M.A.: "General Solutions of the Equations of Elasticity and 
Consolidation for a Porous Material," J. Appl. Mech. (March 1956) 23, 91-96.

27. Muskelishvili, N.I.: "Some Basic Problems of the Mathematical Theory of Elasticity," P. Noordhoff Ltd., Groningen, The Netherlands (1953) 166.

\section{APPENDIX A}

Taking $\sigma_{x x}$ to be negligibly small, the stress/strain relations for an orthotropic material with elastic symmetry about the $x$ and $y$ axes can be written as ${ }^{26}$

$$
\sigma_{y y}=4 N e_{y y}
$$

and

$$
\sigma_{x y}=2 G e_{x y}
$$

These stresses must satisfy the equilibrium equation

$$
\frac{\partial \sigma_{y y}}{\partial y}+\frac{\partial \sigma_{x y}}{\partial x}=0
$$

Using the additional approximation $\partial u / \partial y \ll \partial v / \partial x$ reduces $\mathrm{Eq}$. 1 of the text to

$$
e_{x y}=\frac{1}{2} \frac{\partial v}{\partial x}
$$

Substituting this and the result for $e_{y y}$ from Eq. 1 into the stress/strain relations equation (Eq. A-1) and using the result in the equilibrium equation (Eq. A-2) gives

$$
G \frac{\partial^{2} v}{\partial x^{2}}+4 N \frac{\partial^{2} v}{\partial y^{2}}=0
$$

Letting

$$
y=2 \eta \sqrt{\frac{N}{G}}
$$

reduces Eq. A-4 to Eq. 2 of the text.

\section{APPENDIX B}

We consider the problem that is fundamental to the Griffith crack propagation theory. ${ }^{16}$ This is the problem of the half space $y>0$ as shown in Fig. 11 with the mixed boundary conditions

$$
\begin{aligned}
& \sigma_{x y}=0 \quad \text { for } y=0, \\
& \sigma_{y y}=-p \text { for } y=0,|x|>c,
\end{aligned}
$$

and

$$
v=0 \quad \text { for } y=0,|x|>c .
$$

Sneddon and Elliott ${ }^{19}$ have obtained the exact solution of this problem by means of the theory of elasticity for an isotropic medium. Their solution is

$$
v=\frac{2\left(1-v^{2}\right)}{E} p c \sqrt{1-\left(\frac{x}{c}\right)^{2}} .
$$

We apply to this problem the approximate theory developed in Appendix A. We cannot satisfy all of the boundary conditions at $y=0$, so we choose to allow the condition $\sigma_{x y}=0$ to be violated. The remaining boundary conditions (Eq. B-1) and Laplace's equation (Eq. 2) of the text are verified by means of a conformal transformation. ${ }^{27}$ We consider conformal transformation of the segment $-c<x<c$ onto the unit circle by means of the relation

$$
z=x+i \eta=\frac{c}{2}\left(\zeta+\frac{1}{\zeta}\right)
$$

as illustrated in Fig. 11. A solution of Laplace's equation in the plane

$$
\zeta=r e^{i \theta}
$$

is written in polar coordinates as

$$
\phi=A \frac{\sin \theta}{r} .
$$

This satisfies the boundary condition

$$
\phi=v=0 \text { for } y=0,|x|>c .
$$

The normal derivative on the unit circle and on the corresponding points of the $x$ axis are related as

$$
\left.\frac{\partial \phi}{\partial \eta}=\left|\frac{d \zeta}{d z}\right| \cdot \frac{\partial \phi}{\partial r}\right]_{r=1}=-A\left|\frac{d \zeta}{d z}\right| \sin \theta
$$

On the unit circle we have

$$
\frac{\partial z}{\partial \zeta}=\frac{c}{2}\left(1-\frac{1}{\zeta^{2}}\right)=i c e^{-i \theta} \sin \theta
$$

Hence

$$
\frac{\partial \phi}{\partial \eta}=-\frac{A}{c}
$$

Therefore, from Eqs. 4 and 5, the stress is

$$
p=-\sigma_{y y}=\frac{2 A}{c} \sqrt{N G}
$$

The constant $A$ is determined as

$$
A=\frac{c p}{2 \sqrt{N G}}
$$

The half-width distribution of the crack is

$$
v=\phi(x, 0)=\frac{c p}{2 \sqrt{N G}} \sin \theta=\frac{c p}{2 \sqrt{N G}} \sqrt{1-\left(\frac{x}{c}\right)^{2}}
$$




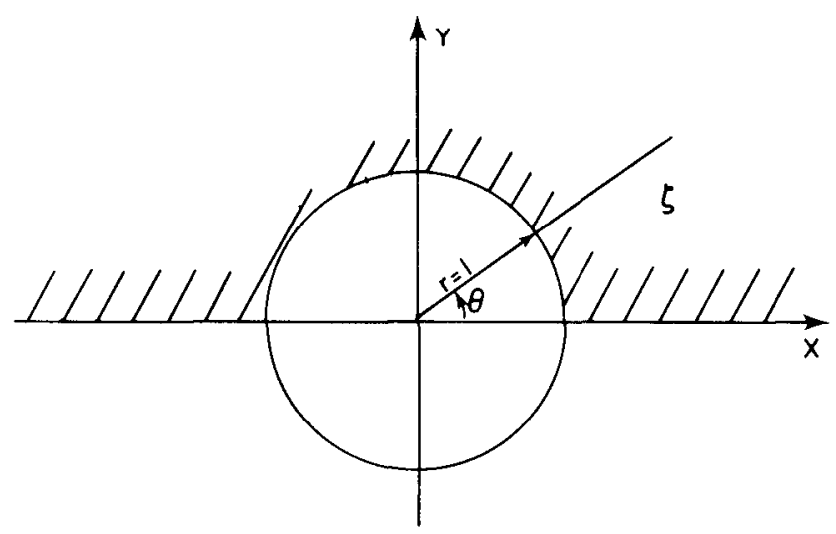

Fig. 11-Conformal transformation of the crack on a unit circle.

Eq. B-12 agrees with the exact result (Eq. B-2) if

$$
2 \sqrt{N G}=\frac{E}{2\left(1-v^{2}\right)} .
$$

This relation is satisfied if we take

$$
G=\frac{E}{2(1+v)}
$$

and

$$
4 N=\frac{E}{2(1-v)\left(1-v^{2}\right)} .
$$

Eq. B-14 identifies $G$ as the shear modulus. In our analysis, $4 N$ is the elastic modulus in plane strain with $\sigma_{x x} \approx 0$. For isotropic media, we should obtain

$$
4 N=\frac{E}{1-v^{2}} .
$$

Thus Eq. B-15 is the correct result when $v=1 / 2$, which corresponds to an incompressible medium. For $v<1 / 2$ Eq. B-15 is not exact, but the error is not large until $v$ becomes small.

\section{APPENDIX C}

Consider a crack of sinusoidal shape in Medium 1. The stress distribution on the crack wall, where $\eta=0$, is also sinusoidal. From Eqs. 4 and 5 of the text,

$$
\sigma_{y y}=-2 \ell \sqrt{N_{1} G_{1}} B \cos \ell x .
$$

The half-width distribution is

$$
v=\phi(x, 0)=B \cos \ell x \text {. }
$$

The elastic energy of the crack is given by

$$
W=-\int_{-c}^{c} \sigma_{y y} v d x=\pi B^{2} \sqrt{N_{1} G_{1}} .
$$

A uniform pressure $p_{1}$ is applied inside the crack. The resulting crack width can be obtained from the principle of virtual work,

$$
\frac{\partial W}{\partial B} \delta B=2 p_{1} \int_{-c}^{c} \delta v d x,
$$

where we have assumed that the crack remains sinusoidal with

$$
\delta v=\delta B \cos \ell x .
$$

\section{This gives}

$$
B-\frac{4 c p_{1}}{\pi^{2} \sqrt{N_{1} G_{1}}}
$$

so the elastic energy becomes

$$
W=\frac{16 c^{2} p_{1}^{2}}{\pi^{3} \sqrt{N_{1} G_{1}}} .
$$

The volume of the crack is

$$
V=2 \int_{-c}^{c} v d x=\frac{32 p_{1} c^{2}}{\pi^{3} \sqrt{N_{1} G_{1}}},
$$

where we have substituted Eqs. C-2 and C- 6 for $v$ and $B$. The condition for crack propagation is obtained from the energy-balance equation

$$
p_{1} \frac{\partial V}{\partial c} \delta c-\frac{\partial W}{\partial c} \delta c=2 S_{1} \delta c .
$$

Substituting for $V$ and $W$ from Eq. C-8 and Eq. C-7 we obtain Eq. 8 of the text.

Eq. 8 can be compared with the result derived by Sneddon and Elliott ${ }^{19}$ from the Griffith theory, ${ }^{16}$

$$
p_{G}^{2}=\frac{2 S_{1} E}{\pi c\left(1-v^{2}\right)}
$$

Substituting Eq. B-13 into Eq. 8 gives

$$
p_{1}^{2}=\frac{\pi^{3} S_{1} E}{32 c\left(1-v^{2}\right)}
$$

This gives

$$
\frac{p_{1}}{p_{G}}=\frac{\pi^{2}}{8}=1.23 \text {. }
$$

Thus the sinusoidal approximation, together with other approximations and assumptions, leads to an error of $23 \%$ in this case. For our purpose, this is not a significant deviation. 


\section{APPENDIX D}

Consider a crack that has penetrated through the interface between Medium 1 and Medium 2 by a distance $b$. Assuming the crack in Medium 2 is approximately triangular in shape, the crack volume is

$$
V=2 \int_{o}^{2 c} v d x+v_{o} b
$$

An approximate relation between $v_{o}$ and $b$ is obtained from Eqs. 10, 15, and 17 of the text and is given by

$$
v_{o}=B \sin 2 \ell c \approx B Z=\frac{\pi b B G_{1}}{2 c G_{2}} .
$$

Substituting this result for $v_{o}$ and Eq. 10 of the text for $v$ into Eq. D-1 and using the approximate relation Eq. 16 of the text for $2 \ell c$ gives

$$
V=\frac{4 B C}{\pi-Z}(1+\cos Z)+B Z b
$$

or, to the first order in $Z$,

$$
V=\frac{8 B c}{\pi}\left(1+\frac{Z}{\pi}\right)
$$

The elastic energy of the crack is given by

$$
W=-\int_{o}^{2 c} v \sigma_{y y} d x
$$

According to Eq. 4 of the text,

$$
\sigma_{y y}=-2 \sqrt{N_{1} G_{1}} B \ell \sin \ell x .
$$

Substituting Eq. D-6 and Eq. 10 of the text into Eq. D-5 gives

$$
W=B^{2} \sqrt{N_{1} G_{1}}\left(2 c \ell-\frac{1}{2} \sin 4 c \ell\right) .
$$

From Eq. 16 of the text,

$$
W=\pi B^{2} \sqrt{N_{1} G_{1}} .
$$

When a fluid pressure $p_{2}$ is applied inside the crack, the virtual work principle gives

$$
\frac{\partial W}{\partial B} \delta B=p_{2} \frac{\partial V}{\partial B} \delta B
$$

Therefore,

$$
B=\frac{4 c p_{2}}{\pi^{2} \sqrt{N_{1} G_{1}}}\left(1+\frac{Z}{\pi}\right) .
$$

Substituting Eq. D-10 into Eq. D-4 and using Eq. 17 of the text for $Z$ gives Eq. 20 of the text. Substituting Eq. D-10 into Eq. D-8 and again using Eq. 17 for $Z$ gives Eq. 21 of the text.

\section{SI Metric Conversion Factor}

$$
\mathrm{lbm} \times 4.535924^{\circ}=\mathrm{kg}
$$

Original manuscript received in Society of Petroleum Engineers office Sept. 1, 1981. Paper accepted for publication Aug. 20, 1982. Revised manuscript received Dec. 7, 1982. Paper (SPE 10372) first presented at the 1981 SPE Eastern Regional Meeting held in Columbus, $\mathrm{OH}$, Nov. 4-6. 